\title{
APPROXIMATE SOLUTIONS FOR COUPLED MOMENT EQUATIONS
}

\author{
GRAHAM WINLEY ${ }^{1}$ and KEITH TOGNETTI ${ }^{2}$
}

(Received 2 February 1981; revised 21 May 1981)

\begin{abstract}
In studying the coupled differential equations for the moments of a stochastic process it is often found that the equation for the $j$ th moment involves higher moments. The usual methods of "decoupling" such a system of equations to obtain estimates of the moments are surveyed and shown generally to result in a system of nonlinear simultaneous differential equations which may be readily solved by numerical methods.

Often, estimates of the first and second moments are the main concern. In this case, two further assumptions reported in the literature can be used to simplify the system and avoid the expense of solving the nonlinear equations. These two techniques are evaluated and compared with a new technique. Two processes are analysed, one representing a chemical reaction and the other population growth.
\end{abstract}

\section{Introduction}

In studying birth and death processes, where the population level at time $t$ is $N(t)$, we consider the system of equations,

$$
\frac{d P_{k}(t)}{d t}=U_{k-1} P_{k-1}(t)+D_{k+1} P_{k+1}(t)-\left(U_{k}+D_{k}\right) P_{k}(t)
$$

where

$$
P_{k}(t)=P\left(N(t)=k / N(0)=N_{0}\right)
$$

and $N(t)$ is confined to a discrete set of states.

\footnotetext{
'Wollongong Institute of Education, Wollongong, N.S.W. 2500.

${ }^{2}$ Department of Mathematics, The University of Wollongong, Box 1144, Wollongong, N.S.W. 2500. Please address any correspondence to the second author at this address.

(1) Copyright Australian Mathematical Society 1982
} 
The quantities $U_{k}$ and $D_{k}$ are called, respectively, the infinitesimal birth and death rates. Further, $U_{k} \Delta t+o(\Delta t)$ is the probability at time $t$ that in a population of size $k$ there will be a single birth in the next time interval $\Delta t$. Similarly $D_{k} \Delta t+o(\Delta t)$ represents the probability of a death in the next time interval $\Delta t$ and $o(y)$ is any function such that $\lim _{y \rightarrow 0} o(y) / y=0$.

The differential equation for the $j$ th moment, $M_{j}(t)=E\left(N^{j}(t)\right)$, can be obtained from (1) by multiplying by $k^{j}$ and summing over $k$ to give

$$
\frac{d M_{j}(t)}{d t}=\sum_{k}\left[U_{k}\left((k+1)^{\jmath}-k^{\jmath}\right)-D_{k}\left(k^{j}-(k-1)^{j}\right)\right] P_{k}(t) \text { for } j \geqslant 1 .
$$

If $U_{k}=\sum_{t=0}^{n} \alpha_{t} k^{i}$ and $D_{k}=\sum_{i=0}^{n} \beta_{t} k^{i}$ we have, from (2), that the differential equation for $M_{j}(t)$ will be free of moments higher than the $j$ th provided $\alpha_{2}=\beta_{2}$ and $\alpha_{i}=\beta_{i}=0$ for $3 \leqslant i \leqslant n$. In this case, the system of differential equations (2) can be solved successively. If this condition is violated we find that the differential equation for $M_{j}(t)$ involves moments higher than the $j$ th and consequently the system of differential equations, an open coupled set, cannot be solved successively. Various methods of decoupling this set of equations have been proposed.

If we are concerned only with obtaining estimates of the first $l$ moments, Goel and Richter-Dyn [2] suggest expressing all the moments higher than the $l$ th moment, appearing in the first $l$ equations, in terms of the first $l$ moments. This is done by using the approximation,

$$
M_{j}(t)=M_{l}(t) M_{j-l}(t) \text { for } j>l .
$$

Wang, [7] assumes that $M_{j}(t) \equiv \xi_{j}^{j}$ is a smooth function of $j$ and uses this to express $\xi_{j}$ for $j>l$, in terms of $\xi_{1}, \xi_{2}, \ldots, \xi_{l}$ by polynomial extrapolation where, in general,

$$
\xi_{j}=\sum_{i=1}^{l}(-1)^{l-i}\left(\begin{array}{c}
j-i-1 \\
l-1
\end{array}\right)\left(\begin{array}{c}
j-1 \\
i-1
\end{array}\right) \xi_{i} \text { for } j>l .
$$

Bailey [1] suggests setting the $(l+1)$ th cumulant of the process to zero. This is equivalent to expressing the $(l+1)$ th moment in terms of lower moments and as with the previous two suggestions results in a set of $l$ nonlinear differential equations which may be solved by numerical methods. Truncation of the set of equations (2) at a higher order results in an improvement of the estimates of the moments but is achieved at the expense of more difficult computation.

If only estimates of $M_{1}(t)$ and $M_{2}(t)$ are required, then McQuarrie [4] suggests two techniques. Each of these avoids the numerical solution of a pair of nonlinear differential equations as required by any of the previous methods. McQuarrie 
presents these two approaches in discussing a stochastic model of an irreversible biomolecular chemical reaction for which results obtained from the exact equations of the process are available for comparison.

We describe a new technique for estimating $M_{1}(t)$ and $M_{2}(t)$ which compares favourably with the two techniques proposed by McQuarrie and produces estimates in closer agreement with the exact solutions. We also consider a biologically plausible model of population growth for which Kendall [3] has shown that the moments cannot be found explicitly by the use of the moment generating function. Applying our techniques, as well as McQuarrie's, to the estimation of the first two moments of this growth process illustrates a serious defect in one of the techniques suggested by McQuarrie.

\section{A system of two coupled equations}

We reduce the system (2) by having $U_{k}=\alpha_{0}+\alpha_{1} k+\alpha_{2} k^{2}, D_{k}=\beta_{0}+\beta_{1} k+$ $\beta_{2} k^{2}$ and $\alpha_{2} \neq \beta_{2}$, to obtain

$$
\frac{d M_{1}(t)}{d t}=c_{0}+c_{1} M_{1}(t)+c_{2} M_{2}(t)
$$

and

$$
\frac{d M_{2}(t)}{d t}=d_{0}+\left(2 c_{0}+d_{1}\right) M_{1}(t)+\left(2 c_{1}+d_{2}\right) M_{2}(t)+2 c_{2} M_{3}(t),
$$

where

$$
c_{t}=\alpha_{t}-\beta_{i} \text { and } d_{t}=\alpha_{t}+\beta_{\imath} \text { for } i=0,1,2 \text {. }
$$

Both the chemical and population processes that we will investigate are in this form. It is necessary to introduce an approximation for $M_{3}(t)$. Following Goel we replace $M_{3}(t)$ in (4) by $M_{1}(t) M_{2}(t)$. Wang's technique replaces $M_{3}(t)$ by $\left(2 \sqrt{M_{2}(t)}-M_{1}(t)\right)^{3}$ and Bailey's suggestion amounts to using $M_{3}(t)=$ $3 M_{1}(t) M_{2}(t)-2 M_{1}^{3}(t)$. Any of these approaches will produce a pair of simultaneous nonlinear differential equations which could be integrated by a numerical method. We will refer to these as the nonlinear techniques.

\section{Fast techniques}

McQuarrie offers two techniques for avoiding the often expensive task of solving these nonlinear differential equations.

In the first technique he uses $M_{2}(t)=M_{1}^{2}(t)$ in (3) to obtain an estimate of $M_{1}(t)$. This estimate of $M_{1}(t)$ together with the assumption that $M_{3}(t)=$ $M_{2}(t) M_{1}(t)$ is then used in (4) to obtain an estimate of $M_{2}(t)$. It is important to 
realise that using $M_{2}(t)=M_{1}^{2}(t)$ in (3) to estimate $M_{1}(t)$ is equivalent to assuming that the variance of the stochastic process is zero only for the purpose of estimating $M_{1}(t)$. This means that the estimate of $M_{1}(t)$ is in agreement with that obtained from the deterministic model of the process. However, the estimate of $M_{2}(t)$ from (4) is not the same as $M_{1}^{2}(t)$. Consequently McQuarrie is not reducing the stochastic model of the deterministic model. In this case, from (3),

$$
M_{1}(t)=\left\{\begin{array}{ll}
\frac{A e^{a t}-B}{2 c_{2}\left[1-C e^{a t}\right]} & \text { for } c_{1}^{2}>4 c_{0} c_{2} \\
\frac{2 N_{0}+\left(N_{0} c_{1}+2 c_{0}\right) t}{2-\left(2 c_{2} N_{0}+c_{1}\right) t} & \text { for } c_{1}^{2}=4 c_{0} c_{2}
\end{array}\right\}
$$

where

$$
\begin{gathered}
a=\sqrt{c_{1}^{2}-4 c_{0} c_{2}}, \quad B=c_{1}-a, \quad D=c_{1}+a, \\
C=\left(2 c_{2} N_{0}+B\right) /\left(2 c_{2} N_{0}+D\right) \quad \text { and } A=D C .
\end{gathered}
$$

Substituting (5) into (4) and using $M_{3}(t)=M_{2}(t) M_{1}(t)$ gives a linear first order differential equation of the form $d M_{2}(t) / d t+P(t) M_{2}(t)=Q(t)$ which can be solved, using the integrating factor $\exp \left[\int P(t) d t\right]$, to give an estimate of $M_{2}(t)$. Equation (5) is a more general result than that proposed by McQuarrie since he considered the use of this technique in relation to (9) and (10), which are special cases of (3) and (4).

To gain insight into the second technique used by McQuarrie we make the simplifying assumption that $M_{2}(t)=f(t) M_{1}^{2}(t)$. Differentiating and using (3) gives

$$
\begin{aligned}
\frac{d M_{2}(t)}{d t} & =f^{\prime}(t) M_{1}^{2}(t)+2 f(t) M_{1}(t) \frac{d M_{1}(t)}{d t} \\
& =f^{\prime}(t) M_{1}^{2}(t)+2 f(t) M_{1}(t)\left[c_{0}+c_{1} M_{1}(t)+c_{2} M_{2}(t)\right] .
\end{aligned}
$$

If we insist that (6) be identical to (4) at $t=0$ then, using $M_{2}(0)=M_{1}^{2}(0)=N_{0}^{2}$, we have

$$
f(0)=1 \text { and } f^{\prime}(0)=\left(d_{0}+d_{1} N_{0}+d_{2} N_{0}^{2}\right) / N_{0}^{2} .
$$

Consequently, we see why McQuarrie assumed that

where

$$
\left.\begin{array}{l}
M_{2}(t)=M_{1}^{2}(t) e^{p t} \\
p=f^{\prime}(0) .
\end{array}\right\}
$$

By using (7) in (3) together with the substitution

$$
M_{1}(t)=-(d u(t) / d t) /\left(c_{2} u(t) e^{p t}\right),
$$


we can obtain the series solution

$$
M_{1}(t)=\frac{c_{2} N_{0}-\sum_{n=2}^{\infty} n u_{n} t^{n-1}}{c_{2}\left[1-c_{2} N_{0} t+\sum_{n=0}^{\infty} u_{n} t^{n}\right] e^{p t}}
$$

where

$$
n(n-1) u_{n}-\left(c_{1}+p\right)(n-1) u_{n-1}+c_{0} c_{2} \sum_{m=0}^{n-2} \frac{u_{m} p^{n-2-m}}{(n-2-m) !}=0 \text { for } n \geqslant 2,
$$

and

$$
u_{0}=1, \quad u_{1}=-c_{2} N_{0} .
$$

Using (8) in (7) gives an estimate for $M_{2}(t)$. It should be noted that implicit in (7) is the assumption that the process has a coefficient of variation (c.v.) given by

$$
(c . v .)^{2}=\left(M_{2}(t)-M_{1}^{2}(t)\right) / M_{1}^{2}(t)=e^{p t}-1 .
$$

For the chemical reaction considered by McQuarrie in (9) and (10) this assumption seems reasonable. However, as we shall see in discussing a population process represented by (12) and (13), this assumption cannot be made for all processes. Again the estimates given by (8) and (7) are more general than those used by McQuarrie who was considering (9) and (10). As we shall see, closed form expressions can often be obtained for $M_{1}(t)$ and $M_{2}(t)$ in (8) and (7).

\section{The new technique $(\operatorname{method} 3)$}

This is another fast technique and, as with McQuarrie's first technique, assumes that $M_{2}(t)=M_{1}^{2}(t)$ in (3) and consequently we arrive at the same estimate of $M_{1}(t)$ as obtained by McQuarrie in (5). This estimate is then used in (4) together with the substitution $M_{3}(t)=3 M_{1}(t) M_{2}(t)-2 M_{1}^{3}(t)$. The result is that (4) becomes a differential equation in $M_{2}(t)$ only. It is different in general from that obtained by McQuarrie's first technique, described above, but is still of a form that can be readily solved analytically by the use of an appropriate integrating factor. McQuarrie's first technique could be described as being based on Goel's suggestion for decoupling (3) and (4) while the present technique is based on Bailey's suggestion where, in both cases, the solution of a pair of nonlinear simultaneous differential equations has been avoided by assuming $M_{1}^{2}(t)=M_{2}(t)$ in (3). 


\section{A chemical process}

In [4], McQuarrie describes an irreversible bimolecular reaction where the differential equations for the moments of the process are given by

$$
\begin{aligned}
& \frac{d M_{1}(t)}{d t}=r M_{1}(t)-r M_{2}(t), \\
& \frac{d M_{2}(t)}{d t}=-2 r M_{1}(t)+4 r M_{2}(t)-2 r M_{3}(t) .
\end{aligned}
$$

It is seen that this is a particular case of (3) and (4).

Using McQuarrie's first technique to decouple (9) and (10) we have, from (5),

$$
M_{1}(t)=\frac{N_{0}}{N_{0}+\left(1-N_{0}\right) e^{-r t}},
$$

which, when used in (10), gives

$$
M_{2}(t)=M_{1}^{2}(t)\left[\frac{2}{3}\left(\frac{N_{0}-1}{N_{0}}\right)\left(e^{2 r t}-e^{-r t}\right)+1\right]
$$

and

$$
(c . v .)^{2}=\frac{2}{3}\left(\frac{N_{0}-1}{N_{0}}\right)\left(e^{2 r t}-e^{-r t}\right) .
$$

Numerical values based on these estimates are shown in Table 1 under the column headed "Method 1". McQuarrie [4] tabulates exact experimental values for this process and these have been reproduced in the column headed "Exact solutions" in Table 1.

If McQuarrie's second technique, which led to the estimates (8) and (7), is applied to (9) and (10) we have

$$
M_{1}(t)=\frac{N_{0}(p+r)}{N_{0} r e^{p t}-\left(r N_{0}-p-r\right) e^{-r t}}
$$

and

$$
M_{2}(t)=M_{1}^{2}(t) e^{p t},
$$

where

$$
p=2 r\left(N_{0}-1\right) / N_{0}
$$

and

$$
(c . v .)^{2}=e^{p t}-1
$$

These estimates have been used to arrive at the numerical values in the column headed "Method 2" of Table 1. 
TABLE 1

Comparison of McQuarrie's techniques (methods 1,2) and the new technique (method 3) with the exact solutions for chemical process

\begin{tabular}{|c|c|c|c|c|c|c|c|c|}
\hline \multirow[b]{2}{*}{$N_{0} r t$} & \multicolumn{2}{|c|}{ Method 1} & \multicolumn{2}{|c|}{ Method 2} & \multicolumn{2}{|c|}{ Method 3} & \multicolumn{2}{|c|}{ Exact solutions } \\
\hline & \multicolumn{2}{|c|}{$M_{1}(t) / N_{0}$} & \multicolumn{2}{|c|}{$M_{1}(t) / N_{0} \quad c}$. & \multicolumn{2}{|c|}{$M_{1}(t) / N_{0}$} & \multicolumn{2}{|c|}{$M_{1}(t) / N_{0} \quad c .1$} \\
\hline & & & & & 10 & & & \\
\hline 0.25 & 0.818 & 0.213 & 0.802 & 0.214 & 0.818 & 0.174 & 0.815 & 0.196 \\
\hline 0.50 & 0.695 & 0.304 & 0.684 & 0.306 & 0.695 & 0.215 & 0.686 & 0.258 \\
\hline 0.75 & 0.606 & 0.375 & 0.592 & 0.377 & 0.606 & 0.240 & 0.593 & 0.300 \\
\hline 1.00 & 0.539 & 0.435 & 0.513 & 0.444 & 0.539 & 0.258 & 0.521 & 0.332 \\
\hline 1.50 & 0.444 & 0.542 & 0.411 & 0.548 & 0.444 & 0.285 & 0.421 & 0.382 \\
\hline & & & & & & & & \\
\hline 0.25 & 0.803 & 0.099 & 0.801 & 0.101 & 0.803 & 0.082 & 0.803 & 0.091 \\
\hline 0.50 & 0.671 & 0.141 & 0.670 & 0.141 & 0.671 & 0.101 & 0.670 & 0.119 \\
\hline 0.75 & 0.577 & 0.172 & 0.575 & 0.172 & 0.577 & 0.113 & 0.576 & 0.137 \\
\hline 1.00 & 0.508 & 0.199 & 0.502 & 0.200 & 0.508 & 0.123 & 0.504 & 0.152 \\
\hline 1.50 & 0.408 & 0.244 & 0.401 & 0.246 & 0.408 & 0.138 & 0.404 & 0.176 \\
\hline & & & & & & & & \\
\hline 0.25 & 0.801 & 0.070 & 0.801 & $0.070^{\circ}$ & 0.801 & 0.075 & 0.801 & 0.059 \\
\hline 0.50 & 0.670 & 0.100 & 0.668 & 0.100 & 0.670 & 0.079 & 0.669 & 0.082 \\
\hline 0.75 & 0.574 & 0.122 & 0.573 & 0.122 & 0.574 & 0.081 & 0.574 & 0.097 \\
\hline 1.0 & 0.503 & 0.141 & 0.501 & 0.141 & 0.503 & 0.087 & 0.502 & 0.109 \\
\hline 1.50 & 0.402 & 0.173 & 0.401 & 0.173 & 0.402 & 0.099 & 0.402 & 0.125 \\
\hline
\end{tabular}

Using our technique (method 3) we have

$$
\begin{aligned}
& M_{1}(t)=\frac{N_{0}}{N_{0}+\left(1-N_{0}\right) e^{-r t}} \\
& M_{2}(t)=\sum_{n=1}^{6} Q_{n} e^{n r t} /\left(e^{r t}+A\right)^{6} \\
& (c . v .)^{2}=\frac{\sum_{n=1}^{6} Q_{n} e^{r t(n-2)}}{\left(e^{n t}+A\right)^{4}}-1
\end{aligned}
$$


TABLE 2

\section{The numerical solutions of the nonlinear pair of}

equations (9) and (10) for chemical process

\begin{tabular}{|c|c|c|c|c|c|c|}
\hline $\begin{array}{l}M_{3}(t) \text { in (10) } \\
\text { replaced by }\end{array}$ & \multicolumn{2}{|c|}{$\begin{array}{c}\text { Goel } \\
M_{1}(t) M_{2}(t)\end{array}$} & \multicolumn{2}{|c|}{ Wang } & \multicolumn{2}{|c|}{ Bailey } \\
\hline$N_{0} r t$ & $M_{1}(t) / l$ & $\mathrm{~V}_{0} \quad c . v$. & $\begin{array}{r}M_{1}(t) / \\
N_{0}\end{array}$ & $\begin{array}{l}v_{0} \quad c . v . \\
=10\end{array}$ & $M_{1}(t) / N$ & $\mathrm{~V}_{0} \quad c . v$. \\
\hline 0.25 & 0.814 & 0.240 & 0.815 & 0.191 & 0.815 & 0.192 \\
\hline 0.50 & 0.680 & 0.382 & 0.687 & 0.250 & 0.687 & 0.255 \\
\hline 0.75 & 0.577 & 0.526 & 0.593 & 0.288 & 0.593 & 0.297 \\
\hline 1.00 & 0.493 & 0.685 & 0.523 & 0.317 & 0.522 & 0.331 \\
\hline 1.25 & 0.422 & 0.871 & 0.476 & 0.340 & 0.466 & 0.360 \\
\hline 1.50 & 0.357 & 1.102 & $\begin{array}{r}0.423 \\
N_{0}\end{array}$ & $\begin{aligned} & 0.359 \\
= & 50\end{aligned}$ & 0.421 & 0.385 \\
\hline 0.25 & 0.803 & 0.111 & 0.803 & 0.091 & 0.803 & 0.089 \\
\hline 0.50 & 0.669 & 0.173 & 0.671 & 0.117 & 0.671 & 0.118 \\
\hline 0.75 & 0.573 & 0.230 & 0.576 & 0.136 & 0.576 & 0.137 \\
\hline 1.00 & 0.499 & 0.287 & 0.504 & 0.151 & 0.504 & 0.152 \\
\hline 1.25 & 0.441 & 0.345 & 0.449 & 0.163 & 0.449 & 0.165 \\
\hline 1.50 & 0.394 & 0.403 & $\begin{array}{r}0.404 \\
N_{0}\end{array}$ & $\begin{aligned} & 0.173 \\
= & 100\end{aligned}$ & 0.404 & 0.176 \\
\hline 0.25 & 0.801 & 0.079 & 0.801 & 0.063 & 0.801 & 0.063 \\
\hline 0.50 & 0.668 & 0.122 & 0.669 & 0.083 & 0.669 & 0.084 \\
\hline 0.75 & 0.572 & 0.162 & 0.573 & 0.097 & 0.573 & 0.097 \\
\hline 1.00 & 0.500 & 0.201 & 0.502 & 0.107 & 0.502 & 0.108 \\
\hline 1.25 & 0.443 & 0.204 & 0.447 & 0.116 & 0.447 & 0.117 \\
\hline 1.50 & 0.397 & 0.279 & 0.492 & 0.124 & 0.402 & 0.125 \\
\hline
\end{tabular}

where

$$
\begin{gathered}
A=\left(1-N_{0}\right) / N_{0}, \quad Q_{1}=2 A^{5} / 3, \quad Q_{2}=5 A^{4}, \quad Q_{3}=16 A^{3}, \\
Q_{4}=\left(N_{0}^{-4}-1-2 A-16 A^{3}-5 A^{4}-2 A^{5} / 3-8 A^{2} r t\right), \\
Q_{5}=2 A \text { and } Q_{6}=1 .
\end{gathered}
$$

Numerical values based on these estimates appear in Table 1 under the heading "Method 3". 
Table 2 shows the numerical solutions of the nonlinear pair of equations (9) and (10), obtained by replacing $M_{3}(t)$ in (10) by the expressions suggested by Goel, Wang and Bailey. A fourth order Runge-Kutta method was used to obtain the solutions.

From Tables 1 and 2 we see that the best estimates of $M_{1}(t)$ and $c . v$. are given by Bailey's method. Wang's technique provides the next best estimates. However, both these techniques, as well as Goel's technique. require the computationally expensive solution of a pair of simultaneous nonlinear differential equations. We note that Goel's technique provides the worst estimates of the seven techniques being compared.

Of the fast techniques (methods 1,2,3) our new technique (method 3) provided an estimate of $M_{1}(t)$ second only to method 2 and gave the best estimate of $c . v$.

\section{A population process}

For the population we define:

$b=$ the mean birth rate per individual at low population level,

$m=$ mean mortality (death rate) per individual at low population level, and $r=b-m$.

Now consider the model described by (1) where

$$
\left.\begin{array}{l}
U_{k}=k b\left[1-\frac{r \gamma_{1} k}{b L}\right] \\
D_{k}=k m\left[1+\frac{r \gamma_{2} k}{m L}\right]
\end{array}\right\}
$$

with

$$
r>0 \text { and } L>N_{0} \geqslant 1 \text {. }
$$

Hence, if the population is of size $k$ at time $t$ then $U_{k} \Delta t+o(\Delta t)$ and $D_{k} \Delta t+o(\Delta t)$ represent the probabilities of a birth and death respectively in the next time interval $\Delta t$. Realistically, these probabilities should depend on $k$, the present population size, and should decrease and increase respectively to the same limiting value as $k$ approaches $L$. Such is the case if we use $U_{k}, D_{k}$ as in (11) with the condition that $\gamma_{1}+\gamma_{2}=1$ where $0 \leqslant \gamma_{1} \leqslant 1$.

Using (11) in (3) and (4) we have

$$
\begin{aligned}
& \frac{d M_{1}(t)}{d t}=r M_{1}(t)-\frac{r}{L} M_{2}(t), \\
& \frac{d M_{2}(t)}{d t}=d_{1} M_{1}(t)+\left(2 r+d_{2}\right) M_{2}(t)-\frac{2 r}{L} M_{3}(t),
\end{aligned}
$$

where $d_{1}=b+m$ and $d_{2}=(r / L)\left(1-2 \gamma_{1}\right)$. 
Decoupling (12) and (13) by McQuarrie's first technique we have

$$
M_{1}(t)=\frac{L}{1+\left(\frac{L-N_{0}}{N_{0}}\right) e^{-r t}},
$$

and

$$
M_{2}(t)=\left\{\begin{array}{c}
\frac{M_{1}^{2}(t)}{L^{2}}\left\{\left[L^{2}+\frac{d_{1} L}{d_{2}}+\frac{L\left(L-N_{0}\right) d_{1}}{N_{0}\left(r+d_{2}\right)}\right] e^{d_{2} t}\right. \\
\left.-\left[\frac{d_{1} L}{d_{2}}+\frac{L d_{1}\left(L-N_{0}\right) e^{-r t}}{N_{0}\left(r+d_{2}\right)}\right]\right\} \text { for } d_{2} \neq 0, \\
M_{1}^{2}(t)\left\{1+\frac{d_{1} t}{L}+\frac{d_{1}\left(L-N_{0}\right)}{N_{0} L r}\left(1-e^{-r t}\right)\right\} \text { for } d_{2}=0
\end{array}\right.
$$

For large values of $t$, these estimates become

$$
M_{1}(t)=L, \quad M_{2}(t)=\left\{\begin{array}{ll}
{\left[L^{2}+\frac{d_{1} L}{d_{2}}+\frac{L d_{1}\left(L-N_{0}\right)}{N_{0}\left(r+d_{2}\right)}\right] e^{d_{2} t}-\frac{d_{1} L}{d_{2}}} & \text { for } d_{2}>0, \\
-\frac{L d_{1}}{d_{2}} & \text { for } d_{2}<0, \\
L d_{1} t+L^{2}+\frac{L d_{1}\left(L-N_{0}\right)}{r N_{0}} & \text { for } d_{2}=0,
\end{array}\right\}
$$

and

$$
(c . v .)^{2}=\left\{\begin{array}{ll}
{\left[1+\frac{d_{1}}{L d_{2}}+\frac{d_{1}\left(L-N_{0}\right)}{L N_{0}\left(r+d_{2}\right)}\right] e^{d_{2} t}-\frac{d_{1}}{L d_{2}}-1} & \text { for } d_{2}>0 \\
-\frac{d_{1}}{L d_{2}}-1 & \text { for } d_{2}<0, \\
\frac{d_{1} t}{L}+\frac{d_{1}\left(L-N_{0}\right)}{r L N_{0}} & \text { for } d_{2}=0 .
\end{array}\right\}
$$

The approximate solution for $M_{1}(t)$ in (24) is recognized as the familiar logistic equation. Since it was obtained by using $M_{2}(t)=M_{1}^{2}(t)$ in (12) we are assuming that the stochastic population model has mean behaviour which is approximated by the deterministic logistic relationship.

$$
\frac{d N(t)}{d t}=r N(t)-\frac{r}{L} N^{2}(t)
$$


The relationship between stochastic models with mean behaviour described by (12) and those with mean behaviour described by (16) is given by Tognetti and Winley [6]. Using our technique for obtaining estimates of $M_{1}(t)$ and $M_{2}(t)$ we have $M_{1}(t)$ as in (14) and

$$
M_{2}(t)=\left\{\begin{array}{l}
\frac{M_{1}^{2}(t)}{L\left(e^{r t}+A\right)^{4}}\left\{\sum_{n=-1}^{4} Q_{n} e^{n r t}+\left[L^{5} N_{0}^{-4}-\sum_{n=-1}^{4} Q_{n}\right] e^{d_{2} t}\right\} \text { for } d_{2} \neq 0, \\
\frac{M_{1}^{2}(t)}{L\left(e^{r t}+A\right)^{4}}\left\{\sum_{n=-1}^{4} R_{n} e^{n r t}+\left[L^{5} N_{0}^{-4}-\sum_{n=1}^{4} R_{n}-\frac{A^{5} d_{1}}{r}\right]\right\} \text { for } d_{2}=0,
\end{array}\right.
$$

where $A=\left(L-N_{0}\right) / N_{0}, \quad Q_{-1}=-A^{5} d_{1} /\left(r+d_{2}\right), \quad Q_{0}=-5 A^{4} d_{1} / d_{2}, \quad Q_{1}=$ $2 A^{3}\left(5 d_{1}+2 r L\right) /\left(r-d_{2}\right), \quad Q_{2}=2 A^{2}\left(5 d_{1}+6 r L\right) /\left(2 r-d_{2}\right), Q_{3}=A\left(5 d_{1}+\right.$ $12 r L) /\left(3 r-d_{2}\right), Q_{4}=\left(d_{1}+4 r L\right) /\left(4 r-d_{2}\right), R_{n}=Q_{n}$, with $d_{2}=0$, for $n=$ $-1,1,2,3,4$ and $R_{0}=5 A^{4} d_{1} t$. For large values of $t$ we have

$$
M_{1}(t)=L, \quad M_{2}(t)=L\left(d_{1}+4 r L\right) /\left(4 r-d_{2}\right)
$$

and

$$
(c . v .)^{2}=\left(d_{1}+L d_{2}\right) / L\left(4 r-d_{2}\right) .
$$

Both techniques assume a mean population size which is approximately logistic. However, our technique leads to a smaller estimate for $M_{2}(t)$ than that obtained by McQuarrie's method.

Turning to the second technique proposed by McQuarrie we assume $M_{2}(t)=$ $M_{1}^{2}(t) e^{p t}$ in (12). Solving for $M_{1}(t)$ we have

$$
M_{1}(t)=\frac{N_{0} L(r+p)}{\left[\left(L(r+p)-r N_{0}\right) e^{-r t}+r N_{0} e^{p t}\right]},
$$

where, from (7),

$$
\begin{gathered}
p=d_{1}+d_{2} N_{0}, \\
M_{2}(t)=M_{1}^{2}(t) e^{d_{1}+d_{2} N_{0}},
\end{gathered}
$$

and

$$
(c . v .)^{2}=e^{d_{1}+d_{2} N_{0}}-1 .
$$

For $0 \leqslant \gamma_{1}<1, p>0$. As $t$ increases, $M_{1}(t)$ increases to a maximum value of

$$
L\left[\frac{p N_{0}}{L(r+p)-r N_{0}}\right] \frac{p}{r+p} \quad \text { at } \quad t=\frac{1}{(r+p)} \ln \left[\frac{L(r+p)-r N_{0}}{p N_{0}}\right]
$$

then decreases asymptotically to zero. 
Pielou [5] discusses the long term behaviour of this population process in the particular case where $b=0.7, m=0.2, \gamma_{1}=0.9, L=100$ and $N_{0}=70$. It is shown that stochastic realizations of the process, obtained by simulation, give a long term mean population size of 99.55 with a variance of 49.13 .

From (15), McQuarrie's first technique estimates the long term population mean at 100 with variance 22500 . From (18) his second technique gives a mean population size and variance which decrease asymptotically to zero. Our new technique (17) predicts a long term population mean of 100 with a variance of 30 .

This indicates that the assumption that $M_{1}^{2}(t) e^{p t}=M_{2}(t)$, which is the basis of McQuarrie's second technique, may not be plausible when "decoupling" moment equations of the type in (3) and (4). On the other hand, we note the reasonable agreement between the results of simulating the process and the estimates obtained by our technique.

\section{Discussion}

A new fast technique for "decoupling" a set of differential equations for the moments of a stochastic process to obtain estimates of the first and second moments has been described. Applied to a stochastic model of a chemical process, the new technique gives estimates which compare favourably with those obtained by more expensive techniques based on the solution of a pair of simultaneous nonlinear differential equations. The new technique is inexpensive and compares most favourably with two other fast techniques proposed by McQuarrie [4]. For the chemical process the new technique provided the second best estimate of the first moment and the best estimate of the coefficient of variation. When compared to results obtained by the simulation of a stochastic population process the new technique gave better estimates of the long term mean and variance of population size than either of McQuarrie's techniques.

\section{References}

[1] N. T. J. Bailey, The elements of stochastic processes with applications to the natural sciences (Wiley, New York, 1964).

[2] N. S. Goel and N. Richter-Dyn, Stochastic models in biology (Academic Press, New York, 1974).

[3] D. G. Kendall, "Stochastic processes and population growth" (Symposium on Stochastic Processes), J. Roy. Stat. Soc. B11 (1949), 230-264.

[4] D. A. McQuarrie, Stochastic approach to chemical kinetics (Methuen, London, 1968).

[5] E. C. Pielou, An introduction to mathematical ecology (Wiley, New York, 1969).

[6] K. Tognetti and G. Winley, "Stochastic growth models with logistic mean population", $J$. Theor. Biol. 82 (1980), 167-169.

[7] Y. K. Wang, "Noise effects and transient behaviour for a laser", Ph.D. Thesis, Yale University, New Haven, Connecticut, 1971. 